\title{
Recurrent proliferating pilar tumour of the scalp mimicking squamous cell carcinoma successfully treated surgically using S-plasty
}

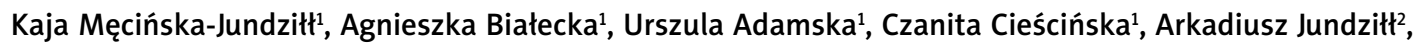 \\ Henryk Witmanowski², Izabela Neska-Długosz ${ }^{3}$, Dariusz Grzanka ${ }^{3}$, Rafał Czajkowski ${ }^{1}$
}

${ }^{1}$ Department of Dermatology and Venereology, Faculty of Medicine in Bydgoszcz, Nicolaus Copernicus University in Torun, Poland ${ }^{2}$ Department of Plastic Surgery, Faculty of Medicine in Bydgoszcz, Nicolaus Copernicus University in Torun, Poland ${ }^{3}$ Department of Clinical Pathomorphology, Faculty of Medicine in Bydgoszcz, Nicolaus Copernicus University in Torun, Poland

Adv Dermatol Allergol 2021; XXXVIII (4): 696-698

DOI: https://doi.org/10.5114/ada.2021.108921

Pilar cysts often occur within the scalp. As they arise from the trichilemma or outer root sheath, they are called "trichilemmal cysts" (TCS). In 1966, Wilson-Jones reported uncommon lesions developing from TCs called proliferating pilar tumours (PPTs) which histologically simulated squamous cell carcinoma [1]. PPTs may develop de novo as well [2]. They usually occur on the scalp with a predilection for women [3]. The histological characteristic is trichilemmal-type keratinization [4]. They may be benign, locally aggressive or malignant [5]. In the literature we can find other terms describing these lesions as subepidermal acanthoma, proliferating trichilemmal cyst, invasive hair matrix tumour, invasive pilomatrixoma, proliferating epidermoid cyst, hydatidiform keratinous cyst, giant hair matrix tumour, trichochlamydoacanthoma or pilar cysts and tumours, what is consistent with their ambiguous biological nature [4-6]. We report a case of recurrent PPT of the scalp mimicking squamous cell carcinoma successfully treated surgically using S-plasty with a satisfying aesthetic effect.

A 58-year-old man was admitted to the Department of Plastic, Reconstructive, and Aesthetic Surgery to treat progressive swelling of the scalp. The lesion appeared 2 years earlier and was treated as an atheroma. The progressive recurrence was observed since then. There was no history of preceding trauma and inflammation. The patient did not suffer from any concomitant diseases. The clinical examination showed firm, erythematous swelling with superficial ulcerations and telangiectasias localized on the scalp (Figure $1 \mathrm{~A}$ ). The lesion had a diameter of approximately $5 \mathrm{~cm}$ and was movable over the underlying bone. Detailed examination did not reveal any relevant abnormalities as lymphadenopathy. Basic labo- ratory tests were normal. The patient was qualified for surgical treatment. The tumour was excised with a $2 \mathrm{~cm}$ margin of normal skin. To reconstruct the large defect of the scalp, given the most satisfying aesthetic effect, S-plasty was used (Figures 1 B-D). The excised solid-cystic nodule was sent for histopathological examination. The lesion manifested microscopically as a large, mainly solid tumour with pushing borders (Figure $2 \mathrm{~A}$ ). A smaller part of the lesion was cystic and resembled a trichilemmal cyst filled with lamellated keratin (Figure 2 B). The solid part consisted of interlacing bands of squamous epithelium (Figure $2 \mathrm{~A}$ ) exhibiting trichilemmal-type keratinization without formation of the granular layer (Figure $2 \mathrm{C}$ ). Focally there was prominent tumour cell atypia and single typical mitoses (Figure 2 D). Overlying epidermis was focally involved by the lesion (Figure $2 \mathrm{E}$ ). The margins were histopathologically clear. Based on the microscopic examination and clinical data, the diagnosis of proliferating pilar tumour was made. The patient is now under 1 year follow-up, and we have not reported any signs of recurrence.

The great majority of PPTs develop within the scalp [7]. There is a distinct predilection for women over the age of 60 [4]. PTTs are usually round subcutaneous swellings with smooth surface but sometimes they may develop ulcerations resembling squamous cell carcinoma (SCC), what is consistent with our patient, or may present as irregular masses [6]. PPTs are usually benign but they may transform into locally aggressive or malignant lesions. The detailed analysis of 76 patients with PPTs conducted by Ye et al. [5] enabled division into three groups: PPTs, low-grade malignant PPTs (LMPPTs) and high-grade malignant PPTs (HMPPTs). The results

Address for correspondence: Kaja Męcińska-Jundziłt, Department of Dermatology, Sexually Transmitted Diseases and Immunodermatology, Faculty of Medicine, Nicolaus Copernicus University, Bydgoszcz, Poland, phone: +48 5258538 67, e-mail: kaja_cm@wp.pl Received: 15.08.2019, accepted: 14.09.2019. 

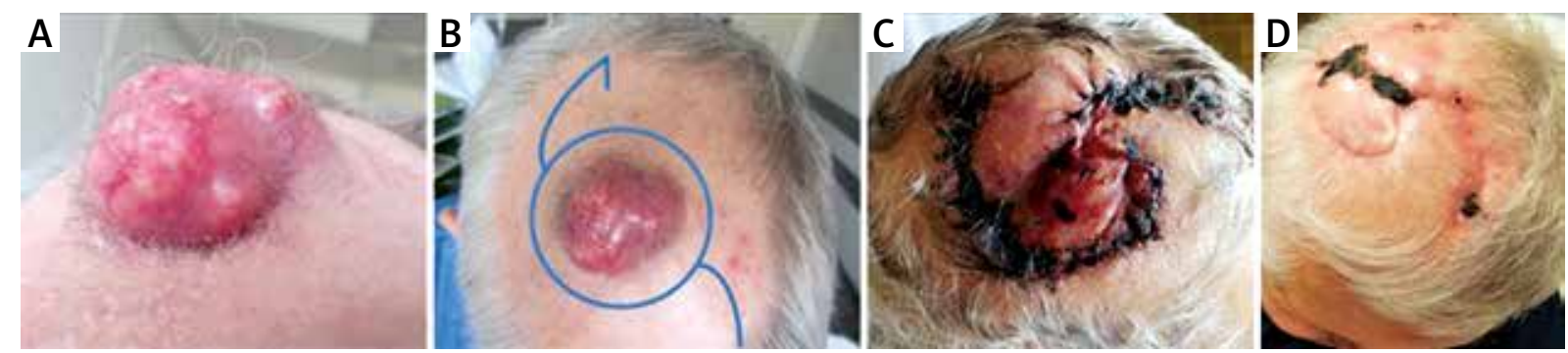

Figure 1. A - Firm, erythematous swelling with superficial ulcerations and extended, branched vessels localized on the scalp. B - S-plasty. Lines of incision. C - Final closure demonstrating S configuration. D - 1 month postoperative
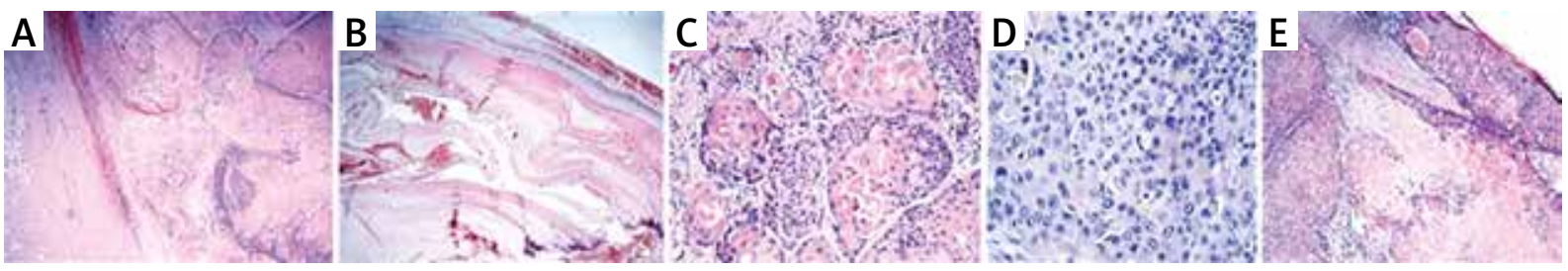

Figure 2. A - Pushing borders of the tumour composed of interlacing bands of squamous epithelium exhibiting trichilemma-type keratinisation. Microscopic slide, HE, 2x. B - Cystic part of the tumour resembling trichilemmal cyst filled with lamellated keratin. Microscopic slide, HE, 2×. C - Trichilemma-type keratinisation without formation of the granular layer. Microscopic slide, HE, 4×. D - Focally prominent nuclear atypia of tumour cells with single typical mitosis. Microscopic slide, HE, 20x. E - Overlying epidermis involved focally by the tumour. Microscopic slide, HE, 2x

showed that PPTs have benign nature, well-defined margins, focal nuclear atypia, typical mitoses, and no vascular or neural invasion. LMPPTs are associated with a small risk of local recurrence, histologically foci of single-cell necrosis are observed and cords of atypical squamous epithelium extend into the surrounding dermis with no marked cytologic atypia. HMPTTs are characterized by cytologic atypia, necrosis, atypical mitoses, and capacity of metastasis development. The main difference between PPTs and LMPPTs or HMPPTs is irregular infiltration of the dermis which may be missed at a surgical excision and may lead to recurrences [5]. This histologic classification is crucial to evaluate the biologic behaviour of the tumour and to determine the range of surgical intervention. Based on clinical aspects and histopathological findings, we classified our case as LMPPT. PPTs show histologic features of SCC as loss of normal epithelium arrangement, differentiation in size and shape of cells and moderate or significant nuclear enlargement, irregularity, and hyperchromatism. The main histologic difference is trichilemmal-type keratinization, present in the case of our patient, in which conversion of the nucleated epithelial cell to anucleate keratinized cell occurs without creation of the granular layer [4]. PPTs are treated surgically by excision. In our patient, we used a $2 \mathrm{~cm}$ margin of normal skin due to prior unsuccessful treatment. Undoubtedly the use of Mohs micrographic surgery may decrease the possibility of recurrences and metastasis.

As the PPTs are usually located within the scalp, there are some aspects to consider relative to surgical treat- ment of this specific area. As the calvarium is three-dimensional, there is limited expandability of scalp tissues, and the aesthetic effect is crucial as it is a hair-bearing region, many options of reconstructive techniques are required. In the case of superficial scalp defects with preserved periosteum, as in our patient, skin grafting and local flaps are most suitable methods even when the defects are large. Due to the low donor site morbidity rate and expectancy of the most satisfying aesthetic outcome, local flaps should be considered as the first-line therapy for the majority of cases. If defects are complex or soft tissues are damaged, due to disabled vascularization, free tissue transfer should be used [8]. Circular excisions provide designated safe margins with minimal waste of skin, but circular defects are usually difficult to close primarily. On the other hand, elliptical excision is an effective surgical technique, but it can lead to skin distortion called "dog ears" and depressed, unaesthetic scars, especially on multidimensional surfaces such as the scalp [9]. In the case of our patient, the decision was made to close the defect using S-plasty as its beneficial results are documented in the literature $[10,11]$. In the first step, margins are drawn around the lesion. Secondly each half of " $\mathrm{S}$ " is drawn inversely to each other. After excision, the wound edges are undermined, rotated in opposite orientations and advanced toward one another [12]. This method decreases the area of removed normal skin and increases the length to width ratio of the incision [13]. The wound tension is better controlled as the distribution of the tension is multidirectional. Use of 
S-plasty reduces the risk of dog ear deformities and enables blending of scars into body contour [9]. There is no focal baldness in contrast to skin grafts. This method is not complex or time-consuming and provides short recovery time with decreased possibility of side effects.

In conclusion, PPTs are interesting lesions with ambiguous biological nature as the clinical features may not correlate with histologic characteristics. Histologic examination is crucial to avoid recurrence and exclude malignancy. PPTs usually develop within the scalp, and reconstruction of the scalp acquired defects remains a great challenge for surgeons. If it is only possible, the simplest methods of reconstruction should be used to achieve the best functional and aesthetic outcome, reduce healing time, and to minimize side effects.

\section{Acknowledgments}

Kaja Męcińska-Jundziłło and Agnieszka Białecka contributed equally to this manuscript.

\section{Conflict of interest}

The authors declare no conflict of interest.

\section{References}

1. Jones EW. Proliferating epidermoid cysts. Arch Dermatol 1966; 94: 11-9.

2. Poiares Baptista A, Garcia E, Silva L, Born MC. Proliferating trichilemmal cyst. J Cutan Pathol 1983; 10: 178-87.

3. Sau P, Graham JH, Helwig EB. Proliferating epithelial cysts. Clinicopathological analysis of 96 cases. J Cutan Pathol 1995; 22: 394-406.

4. Satyaprakash AK, Sheehan DJ, Sangüeza OP. Proliferating trichilemmal tumors: a review of the literature. Dermatol Surg 2007; 33: 1102-8.

5. Ye J, Nappi O, Swanson PE, et al. Proliferating pilar tumors. Am J Clin Pathol 2004; 122: 566-74.

6. Brownstein MH, Arluk DJ. Proliferating trichilemmal cyst: a simulant of squamous cell carcinoma. Cancer 1981; 48: 1207-14.

7. Saida T, Oohara K, Hori Y, Tsuchiya S. Development of a malignant proliferating trichilemmal cyst in a patient with multiple trichilemmal cysts. Dermatologica 1983; 166: 203-8.

8. Steiner D, Hubertus A, Arkudas A, et al. Scalp reconstruction: a 10-year retrospective study. J Craniomaxillofac Surg 2017; 45: 319-24.

9. Kim P, Kim HJ. S-plasty: clinical applications for skin surgery. Aust Fam Physician 2011; 40: 224-5.

10. Mizunuma M, Yanai A, Tsutsumi S, et al. Can dog-ear formation be decreased when an S-shaped skin resection is used instead of a spindle skin resection? A three-dimensional analysis of skin surgery techniques using the finite element method. Plast Reconstr Surg 2000; 106: 845-8.

11. Sebastian S, Bang RH, Padilla RS. A simple approach to the Splasty in cutaneous surgery. Dermatol Surg 2009; 35: 1277-9.

12. Cutaneous Surgery: Illustrated and Practical Approach. Dermatol Surg 1997; 23: 986.

13. Dockery GD. Excisional techniques and procedures. In: Lower Extremity Soft Tissue \& Cutaneous Plastic Surgery. Elsevier 2012; 113-26. 